\title{
Determinación de plomo en lápices labiales de diferentes marcas comercializados en Lima
}

Ángel Alvarado Y.' , Berta Loja H. ${ }^{3}$, Mario Pineda P.3, Miguel Inocente C. ${ }^{3}$, Benjamín Castañeda C.'

\section{RESUMEN:}

Objetivo: Determinar la presencia de plomo en los lápices labiales de diferentes marcas que se expenden en la ciudad de Lima.

Material y Métodos: Se utilizó 24 lápices labiales de cinco marcas comerciales operantes en Perú, procedentes del centro comercial Capón en Lima. La presencia de plomo se determinó por el método de espectrofotometría de absorción atómica con horno de grafito. Para la validación estadística, se determinó la media y la desviación estándar.

Resultados: Se encontró mayor contenido de plomo en las marcas LAL-04-YHBC (3,02 ppm), LAL-05-BALOE (2,10 ppm), LAL02-RW (1,55 ppm) y LAL-03-VCYH (1,22 ppm); contrastada con una marca poco conocida, de código LAL-01-CHOCO PLUSII en la que no se detectó plomo.

Conclusiones: De los lápices labiales estudiados, cuatro de ellos superaron los valores permisibles de plomo (0,1 ppm), que es un valor referencial para caramelos. (Horiz Med 2014; 14(2): 18-21)

Palabras clave: Plomo, lápiz labial, valores permisibles (Fuente: DeCS BIREME)

\section{Determination of Plumb in lip pencils of different commercial marks in Lima}

\begin{abstract}
Objective: To determine the presence of lead in lipsticks from different brands that are sold in the city of Lima.

Material and Methods: 24 lipsticks of five different trademarks were used, from the shopping center in Lima Capon. The presence of lead was determined by the method of atomic absorption spectrophotometry with graphite furnace. For statistical validation, the mean and standard deviation was determined.

Results: The highest lead content was found in the LAL -04 - YHBC (3.02 ppm), LAL -05 - BALOE (2.10 ppm), LAL -02 -RW $(1.55 \mathrm{ppm})$ and LAL -03 marks - VCYH (1.22 ppm ); contrasted with a little known brand, LAL -01- CHOCO PLUSII code where no lead was detected.

Conclusions: Of the studied lipsticks, four exceeded the permissible values of lead $(0.1 \mathrm{ppm})$, which is a reference value for candy. (Horiz Med 2014; 14(2): 18-21)
\end{abstract}

Key words: Lead, lipstick, permissible values. (source: MeSH NLM)

Director del Instituto de Investigación de la FMH de la USMP. Lima-Perú.

Profesor de Química Médica de la FMH de la USMP. Lima-Perú.

Profesores Investigadores del Instituto de Investigación de la FMH de la USMP. Lima-Perú. 


\section{INTRODUCCIÓN}

Un producto cosmético es todo preparado destinado a permanecer en contacto con la superficie del cuerpo humano, con los dientes y con la mucosa bucal, con la finalidad de limpiarlos, perfumarlos, modificar su aspecto, corregir los olores corporales y mantenerlos en buen estado $(1,2)$.

Los lápices labiales son clasificados como cosméticos decorativos, y están en continua renovación, de acuerdo a la tendencia de la moda y la temporada, siendo usado en todos los estratos sociales y desde la pubertad $(3,4)$.

El labio o bermellón, está constituido por fibras musculares estriadas y tejido conectivo fibroelástico; la zona de limbo labial (zona de transición entre la piel y la mucosa) se halla recubierta por un tipo de epitelio con una tonalidad rojiza. Este epitelio, recubre el borde libre del labio, y consta de una capa de células muertas con alto contenido en eleidina (sustancia oleosa formada por lipoproteínas), responsable de su transparencia (5). Debido a su sensibilidad, los lápices labiales deben ser inocuos, ya que si tienen excipientes o ingredientes nocivos, pueden desarrollar desde simples pigmentaciones hasta alteraciones patológicas locales y sistémicas.

El plomo es un elemento metálico, denso de color gris azulado y con brillo metálico, que se ubica en el grupo IV de la Tabla Periódica, con una masa atómica de $270,0 \mathrm{~g} / \mathrm{mol}$, con una densidad de $11,34 \mathrm{~g} / \mathrm{ml}$ y con un punto de ebullición de $1.740^{\circ} \mathrm{C}$.

El plomo puede ser inhalado y absorbido a través del sistema respiratorio ó ingerido y absorbido por el tracto gastrointestinal; la absorción percutánea del plomo inorgánico es mínima, pero el orgánico sí se absorbe bien por esta vía. Una vez absorbido se une a los eritrocitos en un $90 \%$ y presenta modelo farmacocinético tricompartimental, en el compartimento central se halla el $2 \%$ del contenido total, con un tiempo de vida media (t1/2) de 5 semanas, en el segundo compartimento (tejidos blandos) se concentra el $8 \%$, con un t1/2 de 6-8 semanas y en el tercer compartimento (huesos) se concentra el $90 \%$, con tiempo de vida media de 10 a 28 años (6). La eliminación del plomo ingerido se realiza fundamentalmente por las heces, y por la orina $(75 \%)$. Cuando es absorbido por otras vías; faneras (cabello, uñas y sudor) se elimina el $8 \%$. Se elimina por la leche y la saliva, por lo que puede llegar a pigmentar el borde marginal de las encías (Ribete de Burton).

Este metal, no tiene importancia fisiológica; por lo que en el plasma, normalmente, no debería encontrarse. Según la OMS, para considerar una plumbinemia, los valores de plomo deben superar los $15 \mu \mathrm{g} / 100 \mathrm{ml}$; mientras que para el Center of Disease Control (CDC) el nivel de intoxicación es mayor o igual a $10 \mu \mathrm{g} / 100 \mathrm{ml}(7,8)$. Todo producto para el uso 0 el consumo humano, debe tener un registro sanitario, el cual garantiza la calidad e inocuidad de sus principios activos y excipientes.

La evaluación sanitaria de los productos cosméticos, se efectúa de acuerdo al tipo de producto y el riesgo sanitario, para ello se evalúa la etiqueta del producto, los documentos técnicos proporcionados por el fabricante y de ser necesario, se procede a la comprobación del principio activo (9).

El contenido de plomo en lápices labiales, está dado por la cantidad de plomo orgánico (acetato de plomo), contenido en algunos lápices labiales, expresado en partes por millón (ppm) y es cuantificado por espectrofotometría de absorción atómica.

\section{MATERIAL Y MÉTODOS}

La población de estudio estuvo constituida por todos los lápices labiales de la gama de color rojo, de diversas marcas comerciales, que se expenden en el Centro Comercial "Capón" del centro de Lima-Perú. La muestra estuvo conformada por 24 lápices labiales de 5 marcas comerciales.

Fueron las muestras adquiridas en los puestos de ventas, lavadas con solución extra ácida y luego pesadas para obtener $2,0 \mathrm{~g}$ de cada una de ellas para, posteriormente, someterlas a una digestión de microondas durante una hora.

Luego, trasvaso el contenido a un tubo cónico, enrasándolo a $20 \mathrm{ml}$ con agua ultrapura, las mismas 
que luego fueron secadas. El contenido de plomo que se encuentra como plomo orgánico (acetato de plomo) en los lápices labiales, fueron expresados en partes por millón (ppm) y cuantificados por espectrofotometría de absorción atómica con horno de grafito en un Laboratorio de la UNMSM.

\section{RESULTADOS}

Se evaluó cinco marcas comerciales de lápices labiales, de las cuales; cuatro superaron los valores máximos de plomo permitidos, que es de 0,10 ppm, y corresponde a la cantidad de plomo que puede contener un caramelo, según la FDA. Figura 1 y Tabla 1

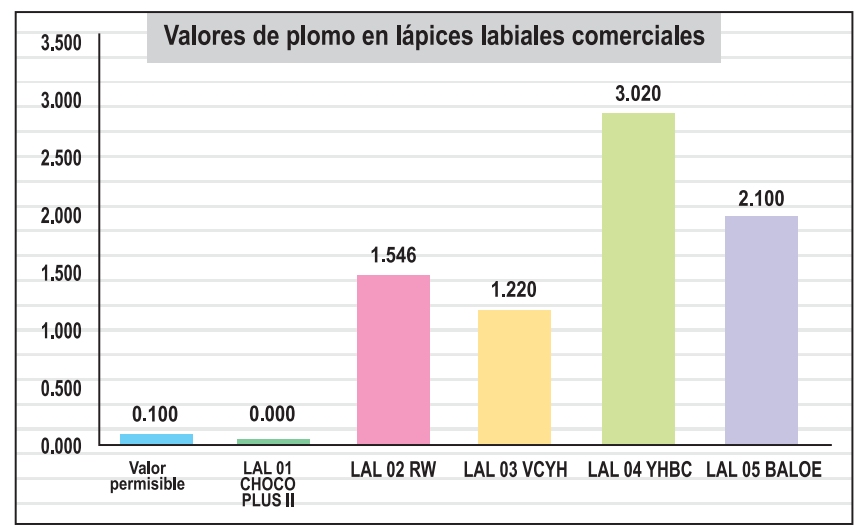

Figura 1. Concentración de plomo en ppm determinados en lápices labiales de la gama de color rojo que se expenden en un Centro Comercial de Lima-Perú

Tabla 1. Valores de plomo determinados por espectrofotometría de absorción atómica en cinco marcas comerciales de lápices labiales

\begin{tabular}{|c|c|c|c|c|c|}
\hline Muestras & $\begin{array}{l}\text { Valores dete } \\
(\mathrm{mg} / \mathrm{L})\end{array}$ & $\begin{array}{l}\text { minados } \\
(\mathrm{ppm})\end{array}$ & $\begin{array}{l}\text { Valor máximo permitido } \\
(\mathrm{ppm})\end{array}$ & $\begin{array}{l}\text { Media } \\
(n=3)\end{array}$ & DS \\
\hline $\begin{array}{l}\text { CHOCOLATE PLUS-II } \\
\text { LAL } 01 \text { CHOCO } \\
\text { PLUS II }\end{array}$ & $\begin{array}{l}00 \mathrm{ppm} \\
00 \mathrm{ppm} \\
00 \mathrm{ppm}\end{array}$ & $\begin{array}{l}00 \mathrm{ppm} \\
00 \mathrm{ppm} \\
00 \mathrm{ppm}\end{array}$ & $0,1 \mathrm{ppm}$ & 00 & 000 \\
\hline $\begin{array}{l}\text { WETNWILD } \\
\text { LAL } 02 \text { RW }\end{array}$ & $\begin{array}{l}1.55 \mathrm{mg} / \mathrm{L} \\
1.54 \mathrm{mg} / \mathrm{L} \\
1.55 \mathrm{mg} / \mathrm{L}\end{array}$ & $\begin{array}{l}1.55 \mathrm{mg} / \mathrm{L} \\
1.54 \mathrm{mg} / \mathrm{L} \\
1.55 \mathrm{mg} / \mathrm{L}\end{array}$ & $0,1 \mathrm{ppm}$ & 1,546 & 0,0058 \\
\hline LABIAL VC - YH & $\begin{array}{l}1.23 \mathrm{mg} / \mathrm{L} \\
1.22 \mathrm{mg} / \mathrm{L} \\
1.22 \mathrm{mg} / \mathrm{L}\end{array}$ & $\begin{array}{l}1.23 \mathrm{mg} / \mathrm{L} \\
1.22 \mathrm{mg} / \mathrm{L} \\
1.22 \mathrm{mg} / \mathrm{L}\end{array}$ & $0,1 \mathrm{ppm}$ & 1,22 & 0,007 \\
\hline BEJA COSMETICS & $\begin{array}{l}3.02 \mathrm{mg} / \mathrm{L} \\
3.02 \mathrm{mg} / \mathrm{L} \\
3.02 \mathrm{mg} / \mathrm{L}\end{array}$ & $\begin{array}{l}3.02 \mathrm{mg} / \mathrm{L} \\
3.02 \mathrm{mg} / \mathrm{L} \\
3.02 \mathrm{mg} / \mathrm{L}\end{array}$ & $0,1 \mathrm{ppm}$ & 3,02 & 0,000 \\
\hline $\begin{array}{l}\text { BELLESPA ALOE } \\
\text { LAL } 05 \text { BALOE }\end{array}$ & $\begin{array}{l}2.10 \mathrm{mg} / \mathrm{L} \\
2.11 \mathrm{mg} / \mathrm{L} \\
2.09 \mathrm{mg} / \mathrm{L}\end{array}$ & $\begin{array}{l}2.10 \mathrm{mg} / \mathrm{L} \\
2.11 \mathrm{mg} / \mathrm{L} \\
2.09 \mathrm{mg} / \mathrm{L}\end{array}$ & $0,1 \mathrm{ppm}$ & 2,10 & 0,010 \\
\hline
\end{tabular}

\section{DISCUSIÓN}

Los resultados obtenidos en el presente trabajo, ponen de manifiesto que el elemento plomo está presente en los lápices labiales que se expenden en Lima. De las cinco marcas comerciales de lápices labiales evaluados el $80 \%$ presentó niveles de concentración de plomo en un rango que va desde 1,22 a 3,02 ppm. En el producto LAL-05-BALOE, se ha encontrado que el contenido de plomo promedio es de 2,10 ppm, con una tendencia a variar por debajo o por encima de dicho contenido en 0,010 ppm (DS): mientras que en el producto LAL-03-
$\mathrm{VCYH}$, se ha encontrado que el contenido de plomo promedio es de 1,22 ppm, con una DS 0,007 ppm; en tanto, el contenido promedio de plomo del LAL-02RW es de 1,546 con una DS de 0,0058 ppm. Siendo el producto con un mayor contenido de plomo el LAL-04-YHBC.

En el 2007, la Campaña para cosméticos seguro (Campaign for Safe Cosmetics) de Estados Unidos, reportó la presencia de plomo en lápices labiales, en un rango de 0,03 a 0,65 ppm en más de 20 muestras de las 33 estudiadas (4). Esto genero controversias y una alerta sobre dicho metal en los cosméticos. 
Mientras que Iman Al-Saleh y cols. (2009), determinaron un nivel de 0,49 a 1,793 ppm de plomo en diversas marcas de lápices labiales y sombras de ojos (10).

Nosotros hemos encontrado que las cuatro marcas superan los valores permisibles que se propone para los caramelos según la FDA $(0,10$ ppm), cuyo valor es referencial y de permisibilidad, ya que hasta la actualidad no se ha legislado sobre el contenido de dicho metal en los labiales. A la vez que las muestras estudiadas por nosotros, superan a los reportados por Iman Al-Saleh.

Estos valores pueden considerarse razonablemente como carcinógenos humanos, pero la Administración de Alimentos y Drogas (FDA) evaluó sobre los posibles daños del plomo en los lápices labiales, determinando que no existe motivo de preocupación debido a que el lápiz labial es para uso local y se ingiere solo por accidente y en cantidades pequeñas (11). Por lo que amerita hacer un estudio con un número mayor de muestras y a la vez involucrar a todas las marcas que se expenden en nuestro país, y realizar exámenes de plomo en las mujeres que usan diariamente, este tipo de cosmético. Asimismo, se debe legislar sobre el contenido de metales pesados en los cosméticos, tal como se ha realizado en Europa, en la que se garantiza la seguridad de cada uno de los ingredientes de los cosméticos (12).

En conclusión, el contenido de plomo, en las muestras estudiadas, se encuentra por encima de los valores permitidos para caramelos como valor de referencia, siendo conveniente establecer valores para productos cosméticos.

\section{Fuentes de financiamiento}

El estudio ha sido autofinanciado por los autores.

\section{Conflicto de interés:}

Los autores declaran no tener ningún conflicto de interés en el trabajo realizado.

\section{REFERENCIAS BIBLIOGRÁFICAS}

1. Reglamento (CE) $N^{\circ} 1223 / 2009$ del Parlamento Europeo y del Consejo. Sobre los productos cosméticos. Diario Oficial de la Unión Europea 2009.

2. Charlet E. Cosmética para farmacéuticos. Zaragoza: Acribia Editorial; 1995.

3. López F, García P. Protección de la piel. Revista Farmacia Hispalense 2011;33:64-4.

4. Bonet R, Garrote A. Cosmética Labial. Offarm 2007;3:26.

5. Azcona L. Cuidado de los labios: hidratación y protección. Revista Farmacia Profesional 2005;1:19:63-4

6. Valdivia M. intoxicación por plomo. Rev. Soc. Per. Med. Inter. 2005;18:1.

7. López J. Intoxicación por Plomo en Niños Menores de Seis Años en un Asentamiento Humano del Callao. Anales de la Facultad de Medicina UNMSM 2000; 1:18.

8. Ascione I. Intoxicación por plomo en pediatría. Arch Pediatr Urug. 2001; 72(2): 133-138.

9. Altunaga L, Yip J et al. Calidad Sanitaria de Cosméticos de producción nacional y de importación durante 1999. Revista Cubana Alimentación y Nutrición 2001;15(1):74-7.

10. Iman Al-Saleh et al. Evaluación de plomo en los productos cosméticos. En Regulatory Toxicology and Pharmacology 2009; 54(2):105-113.

11. Hepp N, Mindak W, Cheng J. Determination of total lead in lipstick: Development and validation of a microwave-assisted digestion, inductively coupled plasma-mass spectrometric method J Cosmet Sci 2009;60:405-414.

12. Vinardell M. Realidad de los métodos alternativos en la evaluación de seguridad de ingredientes cosméticos. Rev Toxicol 2013; 30:3435.

\section{Correspondencia:}

Ángel Alvarado Y.

Dirección: Av. Alameda del corregidor 1531-

Las Viñas, La Molina. Lima - Perú.

Teléfono: 3652300

Correo electrónico: eaa.alvarado@hotmail.com
Recibido: 15 de Enero de 2014 Aprobado: 15 de mayo de 2014 